\title{
Using Content Analysis To Evaluate The Impact of Technological Development In Instructional Materials
}

\author{
Keiler, G.P.; Galembeck, E.
}

\section{Departamento de Bioquímica, IB, Unicamp, SP, Brasil.}

Textbooks are widely used as a teaching tool, and are considered very important in several educational environments, from elementary to tertiary education levels. Since the first textbook arose in the seventeenth century, printed books are the most popular format of textbooks published. New technologies developed over years such as paper, ink and printing hardware. These have made it possible to present printed books in even more attractive formats. Moreover, digital technologies allowed textbooks to provide complementary content, such as software, audio and video. Aiming to understand how new technologies has impacted both textbook presentation and content, we run an external representations (ER) content analysis in a textbook chapter and on an educational software (eBook), both about muscle contraction. The three editions of Bioquímica Básica textbook (1990, 1999 and 2007), written by Anita Marzzoco and Bayardo B. Torres and the four versions of Contração Muscular software developed by Eduardo Galembeck et al. were analyzed. The obtained results showed that the textbook had changes in relation to typography (paper size, kind of paper and colors of printing), and to ERs presented (use of electronic micrograph and 3D models in the $3^{\text {rd }}$ edition and the exclusion of some images and graphs previously used). Regarding the Contração Muscular software, there were several changes in relation to the visual aspect, such as images and icons, and also code updates to make the software compatible with new technologies developed since the first version was released. Although technology has driven some updates in both cases, this happened in different aspects including possibilities to create a modern layout and cheaper printer services, in the case of the textbook, and graphic design and software usability, in the case of the eBook. Thus changing the way information is presented to the users.

Keyword: Biochemistry eBook, Biochemistry textbook, muscle contraction 